\title{
Genome-wide map of RNA degradation kinetics patterns in dendritic cells after LPS stimulation facilitates identification of primary sequence and secondary structure motifs in mRNAs
}

\author{
Yutaro Kumagai ${ }^{1 *}$, Alexis Vandenbon ${ }^{2}$, Shunsuke Teraguchi ${ }^{1 *}$, Shizuo Akira ${ }^{3}$ and Yutaka Suzuki ${ }^{4}$
}

From 15th International Conference On Bioinformatics (INCOB 2016)

Queenstown, Singapore. 21-23 September 2016

\begin{abstract}
Background: Immune cells have to change their gene expression patterns dynamically in response to external stimuli such as lipopolysaccharide (LPS). The gene expression is regulated at multiple steps in eukaryotic cells, in which control of RNA levels at both the transcriptional level and the post-transcriptional level plays important role. Impairment of the control leads to aberrant immune responses such as excessive or impaired production of cytokines. However, genome-wide studies focusing on the post-transcriptional control were relatively rare until recently. Moreover, several RNA cis elements and RNA-binding proteins have been found to be involved in the process, but our general understanding remains poor, partly because identification of regulatory RNA motifs is very challenging in spite of its importance. We took advantage of genome-wide measurement of RNA degradation in combination with estimation of degradation kinetics by qualitative approach, and performed de novo prediction of RNA sequence and structure motifs.
\end{abstract}

Methods: To classify genes by their RNA degradation kinetics, we first measured RNA degradation time course in mouse dendritic cells after LPS stimulation and the time courses were clustered to estimate degradation kinetics and to find patterns in the kinetics. Then genes were clustered by their similarity in degradation kinetics patterns. The 3' UTR sequences of a cluster was subjected to de novo sequence or structure motif prediction.

Results: The quick degradation kinetics was found to be strongly associated with lower gene expression level, immediate regulation (both induction and repression) of gene expression level, and longer 3' UTR length. De novo sequence motif prediction found AU-rich element-like and TTP-binding sequence-like motifs which are enriched in quickly degrading genes. De novo structure motif prediction found a known functional motif, namely stem-loop structure containing sequence bound by RNA-binding protein Roquin and Regnase-1, as well as unknown motifs.

Conclusions: The current study indicated that degradation kinetics patterns lead to classification different from that by gene expression and the differential classification facilitates identification of functional motifs. Identification of novel motif candidates implied post-transcriptional controls different from that by known pairs of RNA-binding protein and RNA motif.

\footnotetext{
*Correspondence: ykumagai@biken.osaka-u.ac.jp; teraguch@ifrec.osaka-u.ac.jp

${ }^{1}$ Quantitative Immunology Research Unit, WPI Immunology Frontier Research

Center, Osaka University, 3-1 Yamada-oka, Suita, Osaka 565-0871, Japan

Full list of author information is available at the end of the article
} 


\section{Background}

The expression of genes is regulated at multiple steps in eukaryotic cells, in which the control of RNA concentrations plays a particularly important role. RNA levels are controlled at both the transcriptional level and the posttranscriptional level. There have been large numbers of genome-wide studies on gene expression dynamics focusing on the transcriptional control such as ImmGen [1] and ENCODE [2] projects, while such studies focusing on the post-transcriptional control were relatively rare until recently [3-8].

Immune cells have to change their gene expression patterns dynamically in response to external stimuli such as pathogen associated molecular patterns (PAMPs), which consist of evolutionally conserved bacterial and viral molecular components, including lipopolysaccharide (LPS) consisting of outer layer of Gram-negative bacteria. Both transcriptional and post-transcriptional controls are important in this response [9]. Especially, changes in posttranscriptional control such as RNA degradation are known to be critical for short-term temporal adaptation of gene expression levels [10]. In macrophages and dendritic cells (DCs) various genes including proinflammatory cytokines such as TNF and IL-6 are induced immediately after stimulation by PAMPs. Those genes are also controlled by post-transcriptional mechanisms involving RNA-binding proteins [11]. Several RNA-binding proteins such as HuR [12], AUF1 [13], Zfp36/TTP [14], Arid5a [15], Rc3h1/ Roquin [16], Zc3h12a/Regnase-1 [17] have been reported to be involved in post-transcriptional regulation of immune gene expression. Typically, such RNA-binding proteins targets sequence and structural motifs in the 3' untranslated region ( $3^{\prime}$ UTR) of RNAs. Deletion of the genes encoding these proteins leads to aberrant immune responses such as excessive or impaired production of cytokines, indicating the importance of such posttranscriptional control.

The next generation sequencing based techniques such as RNA immunoprecipitation sequencing (RIP-seq) or crosslinking and immunoprecipitation sequencing (CLIP-seq) have been used for identification of target motifs of RNA-binding proteins [18]. These methods, which are RNA counterparts of Chromatin immunoprecipitation sequencing (ChIP-seq) to identify cis elements of DNA, however, cannot be used if the RNA-binding protein involved in the process of interest is not known. For DNA cis elements, an alternative approach for identification starts with the classification of genes according to their gene expression pattern [19]. Typically, gene expression time course data after stimulation is obtained, and genes are classified according to the time course by clustering methods. Subsequently, the promoter sequences of genes with similar expression patterns are subjected to de novo motif prediction or detection of known motifs. The advantage of this method is that it does not require any prior information about DNA-binding proteins.

In the current study, we took advantage of the latter approach, starting from classification of genes according to their expression pattern and then leading to de novo motif prediction over a set of sequences, to identify RNA cis elements possibly controlling RNA degradation kinetics. To classify genes by their RNA degradation kinetics, we first measured RNA degradation time course after LPS stimulation. We found a clear tendency in the kinetics depending on the gene expression levels, induction dynamics after stimulation, and the length of 3' UTRs. Genes were, then, clustered by their similarity in degradation kinetics patterns. Degradation patterns resulted in a different clustering genes from that obtained from simply gene expression time course profiles. By applying de novo sequence or structure motif prediction on the resulting clusters of genes we found not only known functional motifs in 3' UTRs, but also novel unknown motifs. Together, the current study indicated that degradation kinetics patterns lead to classification different from that by gene expression and the differential classification facilitates identification of functional motifs.

\section{Methods \\ Cells}

Bone marrow cells were prepared from C57BL/6 female mice, and were cultured in RPMI 1640 supplemented with $10 \%$ of fetal bovine serum under the presence of murine granulocyte/monocyte colony stimulating factor (GM-CSF, purchased from Peprotech) at the concentration of $10 \mathrm{ng} / \mathrm{mL}$. Floating cells were harvested as bonemarrow derived dendritic cells (BM-DCs) after 6 days of culture with changing medium every 2 days. The cells were stimulated with LPS (Salmonella minnessota Re595, purchased from Sigma) at the concentration of $100 \mathrm{ng} / \mathrm{mL}$. At $0,0.5,1,2,3,4,6$, and $8 \mathrm{~h}$ after LPS stimulation, actinomycin D (ActD, from Sigma) was added at the concentration of $10 \mu \mathrm{g} / \mathrm{mL}$. ActD inhibits transcription and therefore RNA molecules in a cell are degraded over time, which can be measured by RNA-seq to obtain degradation kinetics. At $0,0.5,1,2$, and $4 \mathrm{~h}$ after ActD addition (40 samples in total), cells were harvested and lysed by TRIzol (Invitrogen). The lysate was further subjected to RNA isolation according to the manufacturer's instruction. All animal experiments were approved by the Animal Care and Use Committee of the Research Institute for Microbial Diseases, Osaka University, Japan (IFReC-AP-H26-0-1-0).

\section{RNA sequencing}

RNA sequencing was performed as described [20]. The obtained tag sequences were first mapped to rabbit $\mathrm{Hbb2}$ 
gene (added as an internal control) and mouse ribosomal RNAs by using Bowtie 2 [21] and the mapped sequences were removed. The remaining unmapped tags were mapped to mm10 genome by using Tophat 2 [22]. The number of tags mapped to a gene are counted to obtain tag counts for each transcript. Reads per kilobase per million tags (RPKM) values were calculated for each transcript, then averaged for each gene. Quantile normalization was applied to samples of each time points after ActD addition. The tag sequences in FASTQ format were deposited at DDBJ (accession number DRA004766). The full result is shown in Additional file 1.

\section{Pattern classification of degradation kinetics}

Detailed procedure is described in Additional file 2. Briefly, genes having 1 or more RPKMs in at least one time point before adding ActD were selected as "expressed" genes. The time course data of RNA abundance of these expressed genes after ActD addition were used for pattern classification of degradation kinetics. Time courses with less than 20 tags at time 0 before ActD addition were removed. The remaining time course data were then divided into stable and unstable as follows: if the time course has $>89 \%\left(=2^{-4 / 24}\right.$; a level which would correspond with a half-life of $>24 \mathrm{~h}$ ) of the original RNA level at $4 \mathrm{~h}$ after ActD addition it was labeled as long-lived. Other time courses were marked as short-lived. Short-lived time course data were clustered by density peak clustering [23] with arccosine of Pearson correlation coefficient as a distance measure for each time course data. The number of clusters was determined as 16 by visual inspection of the $\gamma$-rank plot, following the guideline in the original paper [23]. For each identified cluster, its degradation rate was calculated by nonlinear fitting [46] to an equation of the form $r(t)=r_{1}+\left(r_{0^{-}} r_{1}\right) \exp (-\delta t)$ with 4 models; $r_{0}=1$ and $r_{1}=0$ with varying $\delta$ (model 1 ); $r_{0}=1$ with varying $r_{1}$ and $\delta$ (model 2); $r_{1}=0$ with varying $r_{O}$ and $\delta$ (model 3 ); varying $r_{0}, r_{1}$, and $\delta$ (model 4). Models were selected according to Akaike's Information criterion (AIC) [24]. The clusters were ranked by the calculated degradation rates. The calculated degradation rate was also used for visualization and categorization of the genes belonging to the cluster.

\section{Classification of genes according to degradation patterns} Genes were clustered according to the patterns detected by the above procedure. Genes have 8 degradation patterns (one for each time point after LPS stimulation), which can consist of one of the 16 short-lived patterns, the long-lived pattern, and/or "not applicable" (NA) which means that degradation time course at the time does not fulfill the conditions for further classification as described above. The distance between each pair of patterns is defined as the distance between the means of the corresponding cluster cores. Density peak clustering with this distance measure was performed, and number of clusters was set to nine clusters after visual inspection of $\gamma$-rank plot. The detailed procedure has been described in Additional file 2.

\section{Gene ontology analysis}

For a given Gene Ontology (GO) term, the number of genes in a cluster or in the whole genome associated with the term were counted. Using the numbers we calculated a hypergeometric $p$ values. Benjamini-Hochberg correction was applied to control false discovery rates (FDRs). Subsequent selection of enriched GO terms were performed based on the calculated $q$ values with threshold $q<0.05$.

\section{De novo sequence motif prediction}

De novo sequence motif prediction was independently performed for each cluster identified according to the degradation pattern. Sequences of 3' UTRs were extracted from the GenBank RefSeq data (as of April 12, 2015). The sequences are cut into bins with length of 100 bases with 50 bases overlap in-between adjacent bins (see Fig. 5a). For the prediction of enriched primary sequence and secondary structure motifs, we prepared at most 700 sets of sequence bins by randomly selecting single sequence bin for each transcript in the cluster. The following analyses were performed on the sets of sequence bins.

i) Primary sequence motif prediction. A de novo sequence motif prediction software Weeder (version 1.4) [25] was run over the sets of sequence bins described above. The position frequency matrices (PFMs) of identified motifs were then converted to position weight matrices (PWMs) with pseudocount 0.8 [26]. Based on the PWMs existence of motifs in 3' UTRs were searched by fimo software from MEME suite (version 4.6.1) [27] with cutoff FDR of 0.05 .

ii) Secondary structure motif prediction. (1) To identify "seeds" of secondary structure motifs, pairwise alignment considering secondary structure was performed by Foldalign software (version 2.5) [28] over the sets of bins of a cluster of interest. (2) From the identified alignments with various lengths and alignment scores, we chose the "seeds" according to the following three criteria for further analysis: (i) having 15 or more bases in length; (ii) whose sequences are evolutionary conserved, namely, averages of phastCons scores (placental 60-way score downloaded from UCSC genome browser) of both sequences are more than 0.7 ; and 
(iii) having scores in the top $0.2 \%$ of the set of alignments of each length. Based on the selected alignments, a stochastic context-free grammar (SCFG) model [29] was built by using Infernal software (version 1.1.1) [30]. (4) Existence of the model in the sequences in the cluster was searched by Infernal, and hits with $E$ value more or equal to 1 were selected and aligned. (5) Based on the new alignments, the SCFG model was updated. This motif build, search, and alignment cycle (3-5), was performed until no novel sequences were selected on the searching step. The resulting SCFG models were used as "motifs", and searched for their existence in 3' UTRs by Infernal with cutoff E value of 1 . Structures of the identified target sequences for motifs were visualized by using RNAfold web server with default parameters and a minimum free energy algorithm (http://rna.tbi.uni vie.ac.at/cgi-bin/RNAWebSuite/RNAfold.cgi) [31].

\section{Over-representation analysis of identified motifs}

Using the number of hits of the motifs in the genome or a cluster of interest, hypergeometric $p$ values were calculated. The calculated $p$ values were further subjected to Benjamini-Hochberg correction to obtain $q$ values. The significance of the over-representation of motifs in a cluster was determined by the $q$ values.

\section{Results}

RNA-seq measurement of genome-wide RNA degradation kinetics in BM-DC on LPS stimulation

To obtain transcriptome-wide RNA degradation kinetics, we used actinomycin D (ActD) chase experiment procedure. Wild-type BM-DCs were stimulated with $100 \mathrm{ng} / \mathrm{mL}$ of LPS for $0,0.5,1,2,3,4,6$, and $8 \mathrm{~h}$, and at each time point ActD was added at the concentration of $10 \mu \mathrm{g} / \mathrm{mL}$. Cells were collected at $0,0.5,1,2$, and $4 \mathrm{~h}$ after the addition of ActD, resulting in $40(=8 \times 5)$ samples in total. Total RNA was prepared and subjected to RNA-seq procedure. The reads were mapped onto mm10 mouse genome, and tag counts and RPKM values for each gene were calculated. This resulted in 13304 genes having 1 or more RPKMs in at least one time point before adding ActD, which we regarded as "expressed" genes.

The procedure to classify the degradation kinetics of each gene in each duration after LPS stimulation is shown in Fig. 1a. ActD is an inhibitor for RNA polymerase II, thus transcription is supposed to be stopped after the addition of ActD and RNAs would undergo degradation over time. Before proceeding to the classification, degradation time courses with less than 20 tags at time 0 before ActD addition were considered as having too few tags for reliable estimation of degradation parameters, and were therefore marked as "not applicable" and removed. In addition, since we were interested in patters of degradation kinetics of quickly degrading genes, the remaining time course data were then divided into longlived and short-lived as follows: if the time course has > $89 \%\left(=2^{-4 / 24}\right.$; a level which would correspond with a half-life of $>24 \mathrm{~h}$ ) of the original RNA level at $4 \mathrm{~h}$ after ActD addition it was labeled as long-lived. As shown in Fig. 1b, this criterion also separated time courses with increasing signals after ActD addition. In our experimental procedure, RNA levels are measured as relative value to whole RNAs. Since, after addition of ActD, short-lived RNAs decrease while long-lived RNAs remain, resulting in long-lived RNAs appearing to have increasing levels.

Under the assumption that transcripts which degraded with similar kinetics might be regulated by a common mechanism, we first clustered the time courses after the addition of ActD of all genes for each time point after LPS stimulation (step 1 in Fig. 1a). The density peak clustering algorithm was applied to the data and the cluster number was determined as 16, as shown in Fig. 1c and Additional file 3. To link the patterns to degradation speed, we performed nonlinear least-square fitting for each cluster (step 2, Fig. 1a). The degradation kinetics of the unstable RNAs in a cluster was modeled by a differential equation $d r / d t=\mu-\delta r$, where $\mu, \delta$ and $r$ represent transcription rate, degradation rate, and RNA level, respectively. The relevant model parameters in each cluster were assessed by Akaike's Information criterion (AIC) as in Methods. The AIC selected the models with incomplete inhibition of transcription for 9 clusters (the model 2 in Methods) and delayed inhibition of transcription for 7 clusters (the model 3 in Methods). Clusters were ranked based on the estimated degradation rates. Cluster images shown in Fig. 1c and Additional file 3 are in the descending order of the rates. Then we assigned the ranks of degradation speed to each time point after LPS stimulation of each gene (step 3, Fig. 1a). The full result was shown in Additional file 4.

We checked the consistency of the estimated degradation kinetics with some representative examples of immune related genes such as $\operatorname{Tnf}$ and $Z f p 36$. Tnf and Zfp36 mRNAs are known to be stabilized transiently at very early time point after LPS stimulation [32]. Our data recapitulated this transient stabilization of the mRNAs. On the other hand, Tnfaip3 did not show change in degradation rate, also matching with former studies where the mRNA showed marginal change in degradation rate after stimulation (Fig. 1d, the upper panels and the middle left panel) [33]. Cytokine Il6 mRNA was destabilized at intermediate time points (3, 4, and $6 \mathrm{~h}$ after LPS stimulation; Fig. 1d, the middle right panel), consistent with former results [34]. We also confirmed that interferon-inducible genes like Stat1 are stable (Fig. 1d, the lowest rows) [35]. Thus, the current 


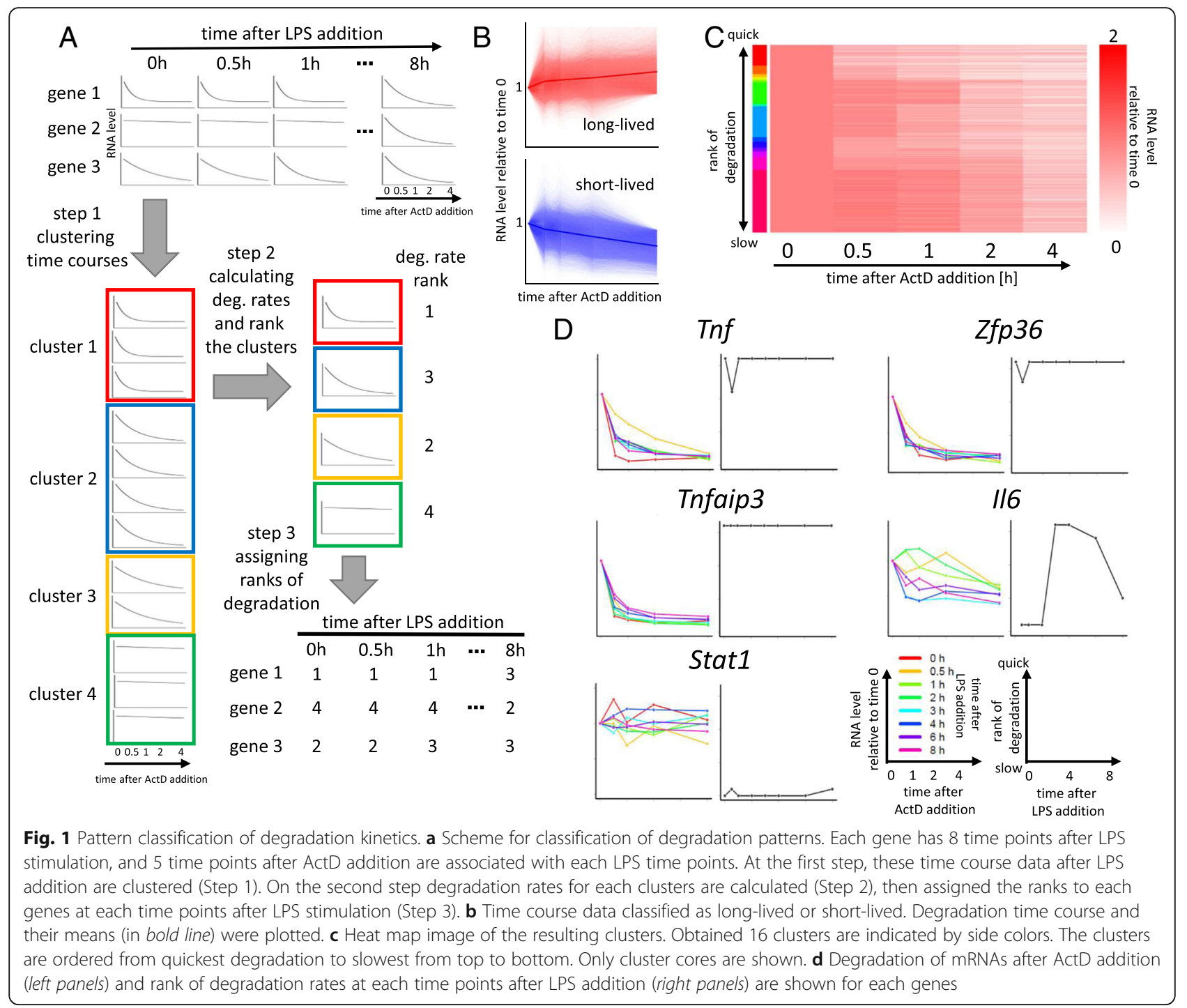

procedure successfully recapitulated known patterns of mRNA degradation kinetics.

\section{Global map of RNA degradation kinetics patterns}

Among the 13304 expressed genes, we focused on 5467 genes whose degradation kinetics were reliably identified in 4 or more out of 8 time points after LPS stimulation (Fig. 2a). The mean degradation speeds of the 5467 genes span from a quarter of hour to $24 \mathrm{~h}$ with median of $3.8 \mathrm{~h}$ in half-life. The 5467 genes were further categorized in the following manner. First, genes labeled as "long-lived" in 4 or more time points after LPS stimulation were categorized as "stable", and remaining genes as "unstable", resulting in 2,398 stable and 3,069 unstable genes, respectively. The distribution of the mean degradation rates of the unstable genes was bimodal (Fig. 2c), which motivated us to further categorize them into two classes. Genes degrading more quickly or more slowly than the threshold 0.325 were categorized as "quick" or "slow", respectively. As shown in Fig. 2a, there were 1629 quick and 1440 slow genes identified.

We next checked how degradation associates with gene expression. Mean gene expression levels were shown in the order of mean degradation rates to compare with each other (Fig. $2 b$ and d). The stable genes tended to have higher gene expression levels than the unstable genes, which was statistically significant as shown in Fig. 2e. Moreover, slow genes had significantly higher gene expression than quick genes (Fig. 2e). Quickly degrading genes and to lesser extent slowly degrading genes were also found to contain significantly higher fraction of upregulated genes than stable genes (Fig. 2f). Also, quickly degrading genes contained higher fraction of upregulated genes than slowly degrading genes did. These results together suggest that quickly degrading genes tend to change their expression level 


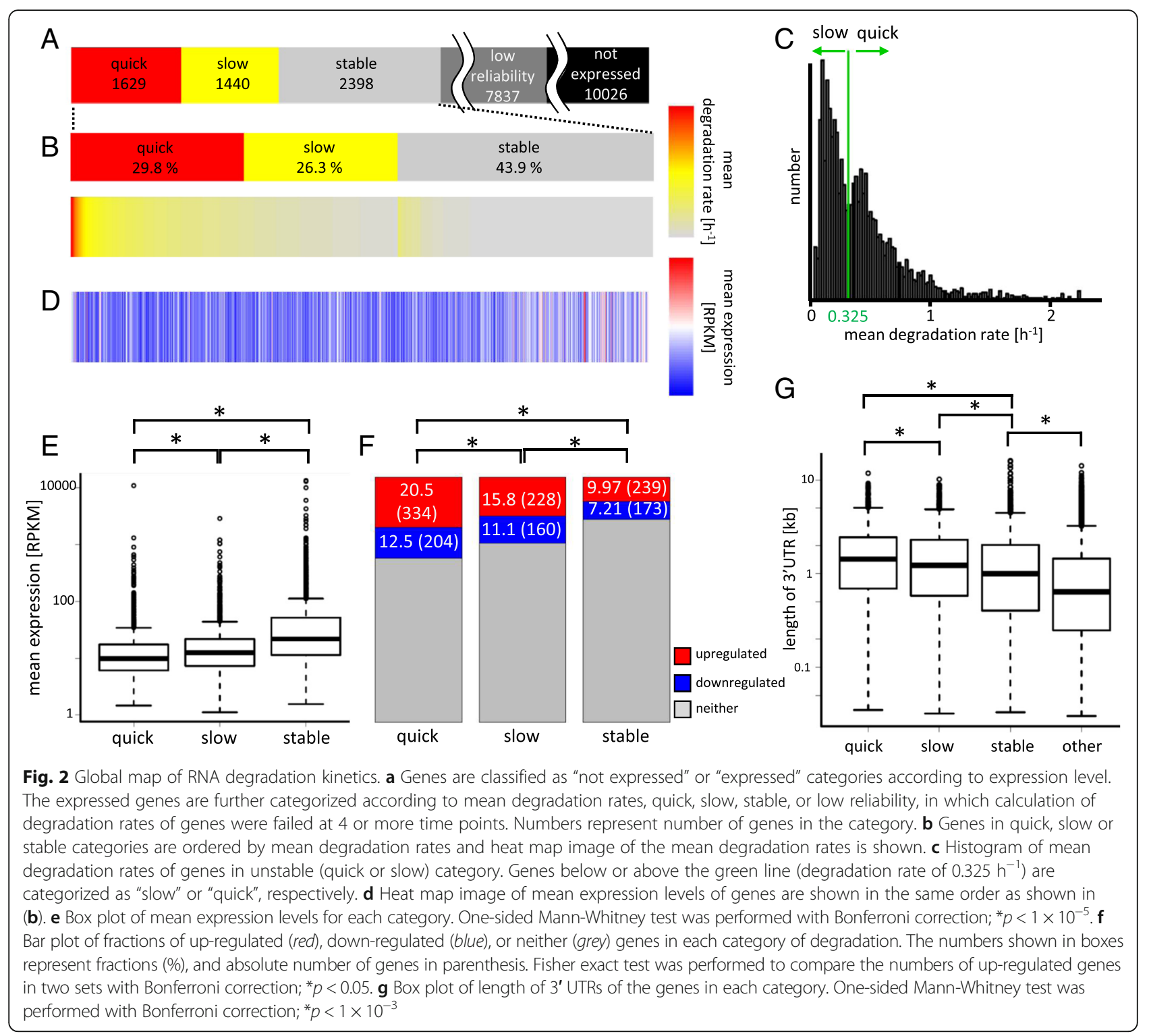

upon stimulation, albeit their mean expression level is lower than others.

The lengths of 3' UTRs in each category were examined as in Fig. 2g. Quickly degrading genes have significantly longer 3' UTRs than slowly degrading, stable, and other genes. Similarly, slowly degrading genes tended to have longer 3' UTRs than stable genes. This implies that quickly degrading genes have longer 3' UTR which may contain larger number of cis elements and may be controlled by multiple mechanisms.

\section{Functional characters of quick, slow, and stable genes}

In order to obtain insights into the functions of genes in each category of genes, we performed GO term enrichment analysis. As shown in Fig. 3, quickly degrading genes tend to be associated with epigenetic controls, as well as IKK/NF- $\kappa B$ signaling. Slowly degrading genes are preferentially associated with GTPase regulation as well as control of RNA splicing. In contrast to preferential association with transcription/post-transcription control of the unstable genes, stable genes were associated with translation and metabolism. This result is consistent with former reports where stable genes are more preferentially linked to translation and metabolic processes [36].

LPS stimulation invokes genome-wide change of gene expression levels. We next asked how degradation kinetics contribute to the change in gene expression level. Genes having more than 3-fold up- or down-regulation were selected, and the up- or down-regulated genes were each further classified into 3 clusters (immediate, early, and late) by $k$-means clustering (Fig. 3b). The number of 


\begin{tabular}{|c|c|c|c|}
\hline & GO_ID & GO_Term & $q$ value \\
\hline \multirow{15}{*}{$\frac{\frac{v}{3}}{\frac{U}{O}}$} & GO:0016568 & chromatin modification & $5.94 \mathrm{E}-11$ \\
\hline & GO:0035097 & histone methyltransferase complex & 3.11E-05 \\
\hline & GO:0045892 & negative regulation of transcription, DNA-templated & 3.75E-05 \\
\hline & GO:0003682 & chromatin binding & 7.83E-05 \\
\hline & GO:0018024 & histone-lysine $\mathrm{N}$-methyltransferase activity & 0.000227 \\
\hline & GO:0018022 & peptidyl-lysine methylation & 0.000242 \\
\hline & GO:0051568 & histone $\mathrm{H} 3-\mathrm{K} 4$ methylation & 0.000551 \\
\hline & GO:0006366 & transcription from RNA polymerase II promoter & 0.001196 \\
\hline & GO:0051091 & $\begin{array}{l}\text { positive regulation of sequence-specific DNA binding } \\
\text { transcription factor activity }\end{array}$ & 0.002141 \\
\hline & GO:0034968 & histone lysine methylation & 0.003077 \\
\hline & GO:0007249 & I-kappaB kinase/NF-kappaB signaling & 0.003077 \\
\hline & GO:0000790 & nuclear chromatin & 0.003169 \\
\hline & GO:0006396 & RNA processing & 0.003417 \\
\hline & GO:0070412 & R-SMAD binding & 0.003489 \\
\hline & GO:0044212 & transcription regulatory region DNA binding & 0.003797 \\
\hline \multirow{15}{*}{$\frac{3}{n}$} & GO:0008060 & ARF GTPase activator activity & 6.67E-05 \\
\hline & GO:0032312 & regulation of ARF GTPase activity & 0.000184 \\
\hline & GO:0005086 & ARF guanyl-nucleotide exchange factor activity & 0.000632 \\
\hline & GO:0071013 & catalytic step 2 spliceosome & 0.000977 \\
\hline & GO:0017111 & nucleoside-triphosphatase activity & 0.001786 \\
\hline & GO:0008380 & RNA splicing & 0.0024 \\
\hline & GO:0006397 & mRNA processing & 0.002995 \\
\hline & GO:0003725 & double-stranded RNA binding & 0.003222 \\
\hline & GO:0007067 & mitosis & 0.003617 \\
\hline & GO:0004386 & helicase activity & 0.003621 \\
\hline & GO:0005681 & spliceosomal complex & 0.004676 \\
\hline & GO:0032012 & regulation of ARF protein signal transduction & 0.005055 \\
\hline & GO:0002199 & zona pellucida receptor complex & 0.005851 \\
\hline & GO:0007050 & cell cycle arrest & 0.008032 \\
\hline & GO:0008026 & ATP-dependent helicase activity & 0.008032 \\
\hline \multirow{14}{*}{$\frac{\frac{\pi}{2}}{\frac{0}{0}}$} & GO:0003735 & structural constituent of ribosome & 2.35E-17 \\
\hline & GO:0022625 & cytosolic large ribosomal subunit & $6.14 \mathrm{E}-15$ \\
\hline & GO:0005764 & lysosome & 2.97E-13 \\
\hline & GO:0005840 & ribosome & $1.73 \mathrm{E}-12$ \\
\hline & GO:0005743 & mitochondrial inner membrane & 4.30E-11 \\
\hline & GO:0030529 & ribonucleoprotein complex & 4.53E-11 \\
\hline & GO:0009986 & cell surface & $3.10 \mathrm{E}-10$ \\
\hline & GO:0022627 & cytosolic small ribosomal subunit & $4.72 \mathrm{E}-10$ \\
\hline & GO:0030246 & carbohydrate binding & 2.90E-09 \\
\hline & GO:0005975 & carbohydrate metabolic process & 3.47E-09 \\
\hline & GO:0006412 & translation & 4.52E-09 \\
\hline & GO:0015935 & small ribosomal subunit & 3.06E-07 \\
\hline & GO:0006629 & lipid metabolic process & 5.44E-07 \\
\hline & GO:0004553 & hydrolase activity, hydrolyzing O-glycosyl compounds & 7.06E-07 \\
\hline
\end{tabular}
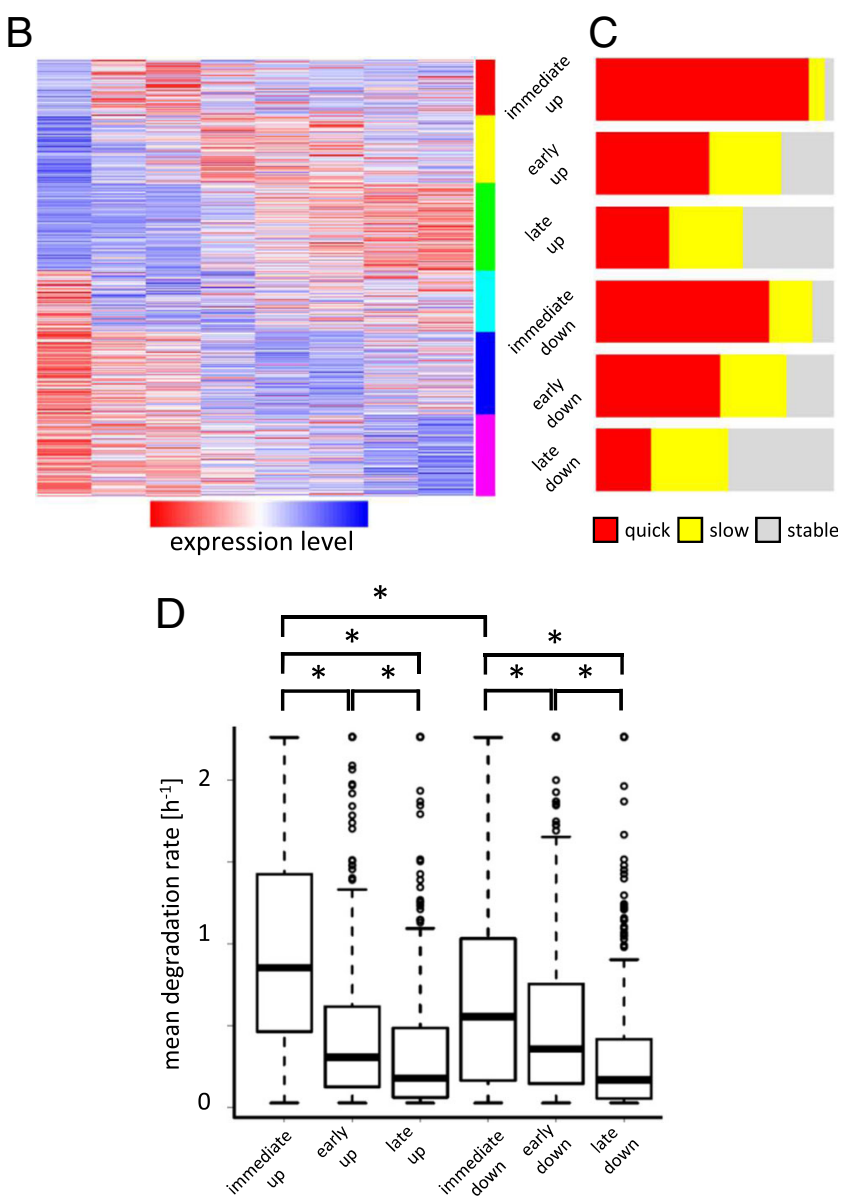

Fig. 3 Functional characters of quick, slow, and stable genes. a Result of Gene Ontology term enrichment analysis on quick, slow, and stable genes. b Heat map image of clusters based on gene expression time course data. The clusters were labeled as "immediate up", "early up", "late up", "immediate down", "early down", or "late down" by visual inspection. c Bar plot of fraction of quick (red), slow (yellow) or stable (grey) genes in each clusters. $\mathbf{d}$ Box plot of mean degradation rates of genes in each clusters. One-sided Mann-Whitney test was performed; ${ }^{*} p<1 \times 10^{-3}$

quickly and slowly degrading or stable genes in each cluster were counted (Fig. 3c). Interestingly, sets of immediately up- or down-regulated genes tend to have significantly larger number of quickly degrading genes than the others $\left(p<1 \times 10^{-4}\right.$, Fisher exact test with Bonferroni correction), whereas comparison between immediate upregulated gene set and down-regulated gene set has no significant change $(p>0.05)$. However, mean degradation rate was significantly higher in immediately up-regulated gene set than in immediately early down-regulated one (Fig. 3d). These results collectively suggested that quick degradation kinetics strongly associated with immediate regulation (both induction and repression) of gene expression level.

\section{Clusters of genes shared patterns in change of degradation rates over time}

De novo search of DNA cis element is often carried out over a set of genes clustered according to their gene expression time course [19]. This is based on the assumption that the time course represents the change of activity of DNA cis elements which control genes in the cluster. For de novo identification of RNA cis element based on the similar assumption, we first clustered transcripts according to time course of degradation patterns found above.

We separated genes having more than 4 "long-lived" time points as stable genes as described above. From the remaining 3067 unstable genes, the density peak clustering gave nine clusters (Fig. 4a and Additional file 2). Together with transcripts labeled as stable, this resulted in 10 clusters (full result is in Additional file 4). The clusters were further ordered by mean degradation rates of genes in the clusters, from quick to slow, and named as Cluster I, II, ... IX, and stable (Fig. 4a). The clusters contain more than hundreds of genes with exception of Clusters IV and IX, containing small number of genes. We excluded these two clusters from further analysis.

First, since we have shown that degradation is associated with gene expression level, we compared the mean 


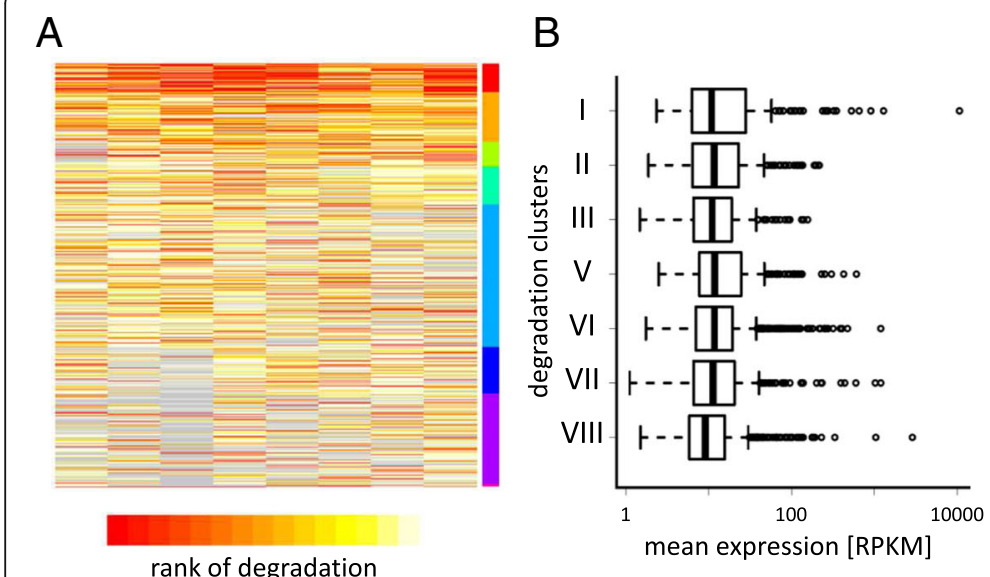

rank of degradation

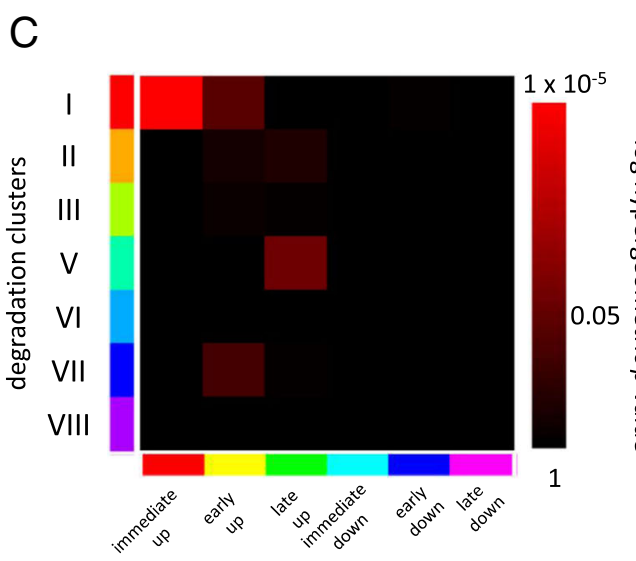

expression clusters
$\mathrm{D}$

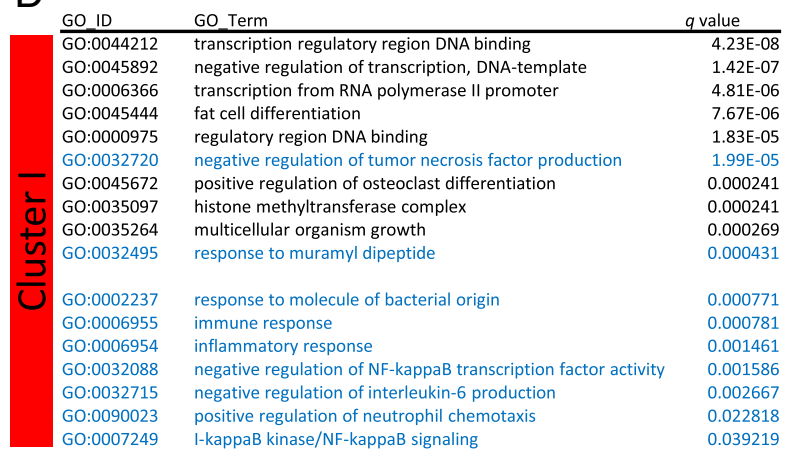

$\mathrm{E}$

\begin{tabular}{|c|c|c|}
\hline GO ID & GO Term & $q$ value \\
\hline GO:0016568 & chromatin modification & $4.56 \mathrm{E}-10$ \\
\hline GO:0004842 & ubiquitin-protein ligase activity & $2.92 \mathrm{E}-06$ \\
\hline GO:0045892 & negative regulation of transcription, DNA-templated & $5.40 \mathrm{E}-06$ \\
\hline GO:0042127 & regulation of cell proliferation & $1.66 \mathrm{E}-05$ \\
\hline GO:0016874 & ligase activity & 6.77E-05 \\
\hline GO:0006357 & $\begin{array}{l}\text { regulation of transcription from RNA polymerase II } \\
\text { promoter }\end{array}$ & $7.88 \mathrm{E}-05$ \\
\hline GO:0015630 & microtubule cytoskeleton & 8.13E-05 \\
\hline GO:0003682 & chromatin binding & 0.000102 \\
\hline GO:0016605 & PML body & 0.000275 \\
\hline GO:0051213 & dioxygenase activity & 0.000485 \\
\hline GO:0034097 & response to cytokine & 0.000608 \\
\hline GO:0042832 & defense response to protozoan & 0.002302 \\
\hline GO:0042102 & positive regulation of $T$ cell proliferation & 0.005031 \\
\hline GO:0007249 & I-kappaB kinase/NF-kappaB signaling & 0.018939 \\
\hline GO:0042110 & T cell activation & 0.034382 \\
\hline GO:0045087 & innate immune response & 0.048596 \\
\hline
\end{tabular}

Fig. 4 Clustering of genes based on degradation patterns. a Heat map image of clusters of genes based on degradation patterns. Color codes represent rank of degradation patterns. Grey represents "NA". b Box plot of mean gene expression levels of genes in each degradation pattern-based cluster (a). No significant differences were observed by two-sided Mann-Whitney test with Bonferroni correction $(p>0.05)$, except for cluster VIII compared to clusters I, II, V, VI, or VI ( $p<0.05)$ (c) Overlaps between the degradation pattern-based clusters (a) and the gene expression-based clusters shown in Fig. 3b. Hypergeometric $p$ values of overlaps are shown. d, e Gene Ontology term enrichment in clusters I (d) and II (e). GO terms in blue letters represent immunity-related ones. Top $10 \mathrm{GO}$ terms and significant $(q<0.05)$ immunity-related terms are shown

expression levels of genes in each cluster. As shown in Fig. 4b, mean gene expression levels were comparable between all clusters, without significant differences $(p>0.05$, after Bonferroni correction), except for cluster VIII compared to clusters I, II, V, VI, or VI $(p<0.05)$. This indicated that although mean degradation rate is strongly associated with mean expression level, the classification based on the degradation kinetic patterns gives unique clustering of genes different from that on gene expression level. The clusters were further compared to gene expression change-based clusters obtained in Fig. 3b. Hypergeometric $p$ values of overlap between two clusters were calculated and shown in Fig. 4c. Cluster I, which contains large number of quickly degrading genes, has significant overlap with immediately up-regulated gene set $\left(p<3 \times 10^{-5}\right)$, further supporting quickly degrading genes are more likely to be up-regulated at early time point after LPS stimulation. The other significant overlap was found only between Cluster V and late up-regulated gene $(p<0.05)$, suggesting that time course of change in degradation pattern offers different classification than gene expression time course or mean degradation rate does.

Cluster I contains numbers of well-known quickly degrading genes such as Tnf, Zfp36, Tnfaip3and Nfkbia [35]. Gene Ontology term enrichment analysis showed that the cluster is associated with transcriptional regulation and immune responses (Fig. 4d). The cluster also contains Ppp1r10, Ier3 and Nfkbid as well as Tnf, known targets for RNA-binding proteins Roquin and Regnase-1, implying transcripts in the cluster are regulated by similar mechanism involving Roquin and Regnase-1 [37, 38].

Cluster II is preferentially associated with chromatin modification genes such as Kdm6b and Setdb1, ubiquitin ligases, and to lesser extent immune response genes, such as Il6, Nfkbiz, Nfkb1, and Irak3. Il6 and Nfkbiz are also known targets for Roquin and Regnase-1 [37, 38], suggesting genes targeted by same RNA-binding proteins do not necessarily share a pattern of degradation kinetics, and regulatory mechanism of degradation of the genes in the cluster is rather heterogeneous (Fig. 4e). 
De novo prediction of primary sequence motifs in 3' UTRs Stability of mRNAs is thought to be controlled largely through their 3' UTRs. Indeed, several primary sequence motifs in 3' UTR, such as AU-rich elements (AREs) [39], are known to control mRNA stability. Thus it is natural to apply de novo prediction of sequence motifs to 3' UTR sequences of genes in a cluster thought to be controlled by similar degradation mechanism. To test if the current classification of genes based on degradation kinetics patterns helps the identification of sequence and secondary structure motifs de novo, we first searched for primary sequence motifs on 3 ' UTRs of the transcripts in Cluster I obtained above, since this cluster is enriched for immune related genes. The procedure is shown in Fig. 5a. First we cut the 3' UTR sequences into 100 base long bins with 50 bp overlap between adjacent bins. Those bins were randomly picked from each gene to make a set of 3' UTR sequence bins in the cluster. Sets were randomly generated for hundreds of times, and on each set we used the de novo sequence motif prediction program Weeder. After identifying motif candidates, their over-representation in the cluster was assessed by calculating FDRs.

A list of enriched motifs found in Clusters I is shown in Fig. 5b and c, respectively. The identified motifs were largely similar to each other. $\operatorname{Tnf}, Z f p 36$, and other genes are known to be regulated through AREs in their 3' UTRs [39]. Typical ARE has AUUUA pentamer, with some variation in franking nucleotides [40]. As expected from the presence of $T n f$ and $Z f p 36$ in the cluster, the enriched motifs in the cluster are abundant in $U$ and to lesser extent A. Furthermore, some of the motifs such as motif.109.8 and 42.17 contain AUUUA-like stretch (Fig. 5c), fitting with the consensus motif of AREs. Moreover, these motifs resemble typical TTP binding motif UAUUUAU [41], suggesting the control on

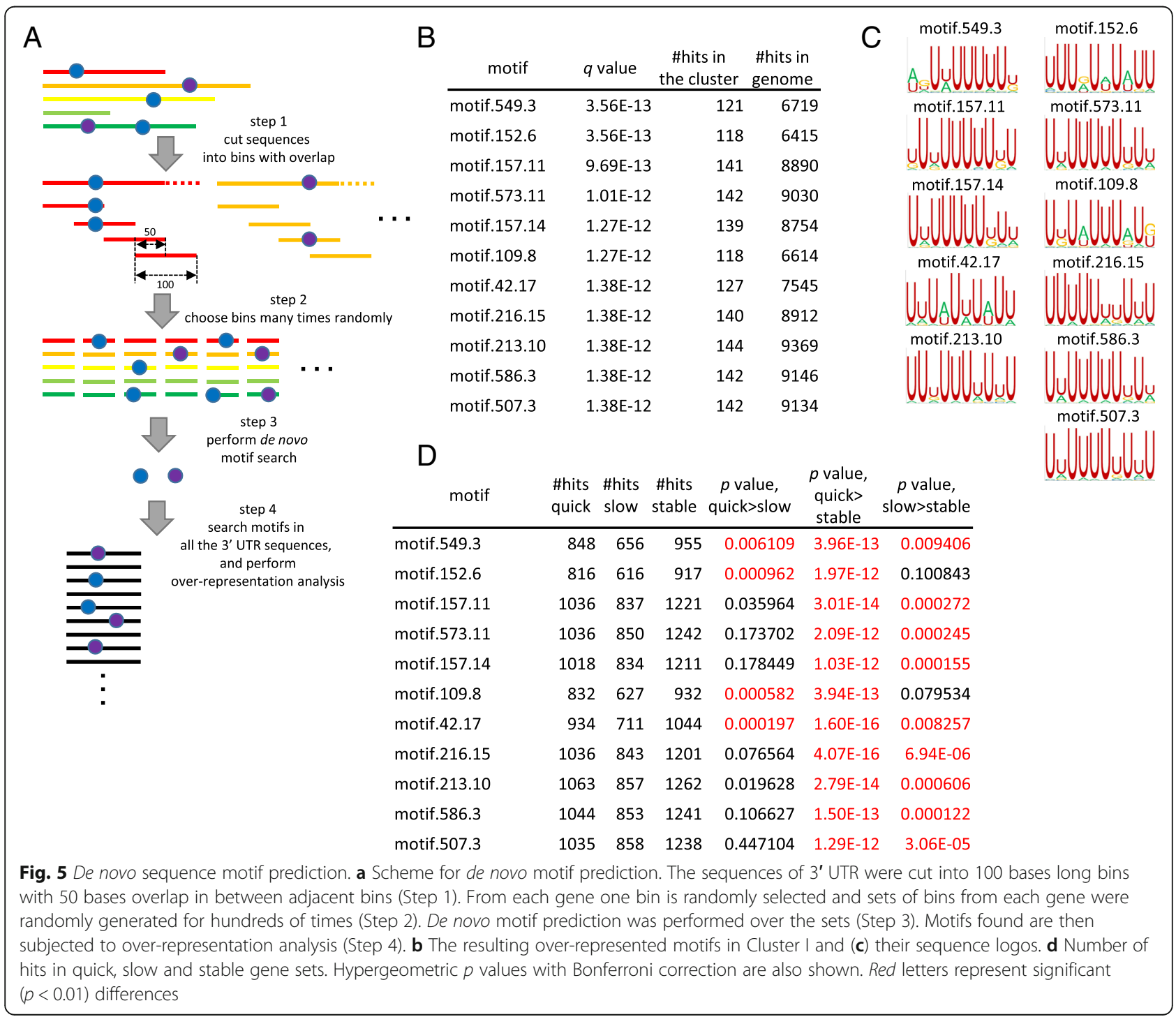


number of genes in the cluster by TTP. Finally, we checked the number of presences of these motifs in quick, slow or stable gene sets, and found that the set of quickly degrading genes has significantly higher numbers of instances than the sets of slowly degrading genes or stable genes (Fig. $5 \mathrm{~d}$ ). Collectively, de novo prediction of sequence motifs in genes sharing the same degradation pattern identified ARE-like motifs, which are abundant in 3' UTR of quickly degrading genes.

\section{De novo prediction of secondary structure motifs in $3^{\prime}$ UTRs}

Unlike DNA, most cellular RNAs are single-stranded and form secondary structure, such as stem loop structure. Typical examples are transfer RNA and ribosomal RNAs, which form large secondary structure, and form complex with proteins. For the interaction between RNAs and proteins, such secondary structures are essential. Previous studies have identified various secondary structure motifs bound by certain RNA binding proteins, such as above mentioned RNA-binding proteins [37, 38, 41-43]. Their identification, however, has been based on the prior knowledge that particular RNAs are targets of a particular RNA-binding protein. Since there are hundreds of RNA-binding proteins, it is favorable to identify RNA motifs even when there is no such prior knowledge. Thus, we further sought to identify secondary structure motifs enriched in the cluster in addition to the sequence motifs.

Several methods to extract secondary structure motifs from a set of transcript sequences have been proposed. Some studies took advantage of pairwise or multiple alignment of RNA sequences considering both sequence similarity and secondary structure [43, 44]. We utilized a similar approach with modifications to detect motifs that are enriched in a cluster of interest, but not necessarily form a majority in the cluster. The latter point is crucial since we are not starting from a set of transcripts identified as targets of a common RNA-binding protein, but from a set of transcripts which share only degradation kinetics.

The procedure is briefly depicted in Fig. 6a. We first carried out pairwise sequence and structure alignment of RNAs on 3' UTR sequence bins. From Cluster I, over 6 million of alignments were found, which are regarded as "seeds" for motifs. We checked the distribution of scores and lengths of the alignments (Additional file 5) and found that they are not independent. Thus, we first divided the alignments into sets of the same length. Then motif seeds were selected from each sets with the following criteria: (1) Aligned sequences are conserved, namely, have more than 0.7 mean phastCons score, and (2) alignment score in the top $0.2 \%$ of the set with the same alignment length. After this selection we retained 679 motif seeds from this cluster. Stochastic context free grammar (SCFG) models [29] were built from the selected motif seeds as described in the Methods, and over-representation in the cluster was checked. As shown in Fig. 6b, we obtained 13 motifs with less than $1.0 \times 10^{-4} \mathrm{FDR}$ and 10 or more instances in the cluster. Roughly, they were divided into 3 different motifs (red, black and blue in Fig. 6b). All the alignments with common structure for motif.0.271, motif.0.170, and motif.0.107 are shown in Additional file 6. Among the motifs, motifs $0.271,0.34,0.310,0.236$ and 0.551 were found to be similar to the stem-loop motif bound by Roquin- or Regnase-1 (Fig. 6b, in red). As shown in Fig. 6c, Tnf, Ier3, Ppp1r10 and Nfkbid were found to contain the motif, and the stem loops are almost identical to that of Roquin or Regnase-1 targets [37, 38]. Other members in the Cluster I, such as Ptger4, Iffo2, Tagap, as well as known Roquin or Regnase-1 targets in other clusters such as Nfkbiz and Roquin, also have the stem loop. This indicates that the current procedure in combination with genome-wide measurement of RNA degradation kinetics successfully identified functional secondary structure RNA motif without prior information.

In addition to this known functional motif, other stem loop motifs were identified in the current cluster. Motifs indicated in black in Fig. 6b, including motif.0.107 and 0.580, have many AU repeats (Additional file 6), and it is not clear if they represent functional protein binding motifs. Motif.0.170, 0.199, and 0.379 (shown in blue letters in Fig. 6b), on the other hand, were not identical to the Roquin-binding stem loop and did not have repeat sequences. As shown in Fig. 6d, Tnf, Zfp592, Btg2, and Socs3, contain this stem loop structure. We checked GO term enrichment for genes having motif.0.271 or motif.0.170 (tables in Fig. 6c and d, respectively), and found genes with motif.0.271, Roquin and Regnase-1 target, were associated with terms such as $\mathrm{T}$ cell proliferation, while those with motif.0.170 were preferentially associated with terms such as JAK-STAT cascade, further implying the role of those motifs in immune responses. These results collectively suggest that degradation patterns of genes in this cluster is regulated by mechanisms which are different from those controlled by Roquin and Regnase-1, and involve the binding of a regulator to this stem-loop structure.

\section{Discussion}

In the current study we conducted genome-wide measurement of RNA degradation kinetics, and used the dynamical pattern in degradation rates for predicting sequence and structure motifs of genes under the similar regulatory mechanism. A number of genome-wide RNA degradation measurements have been reported before [3-8]. Despite cell types and methods being differed 


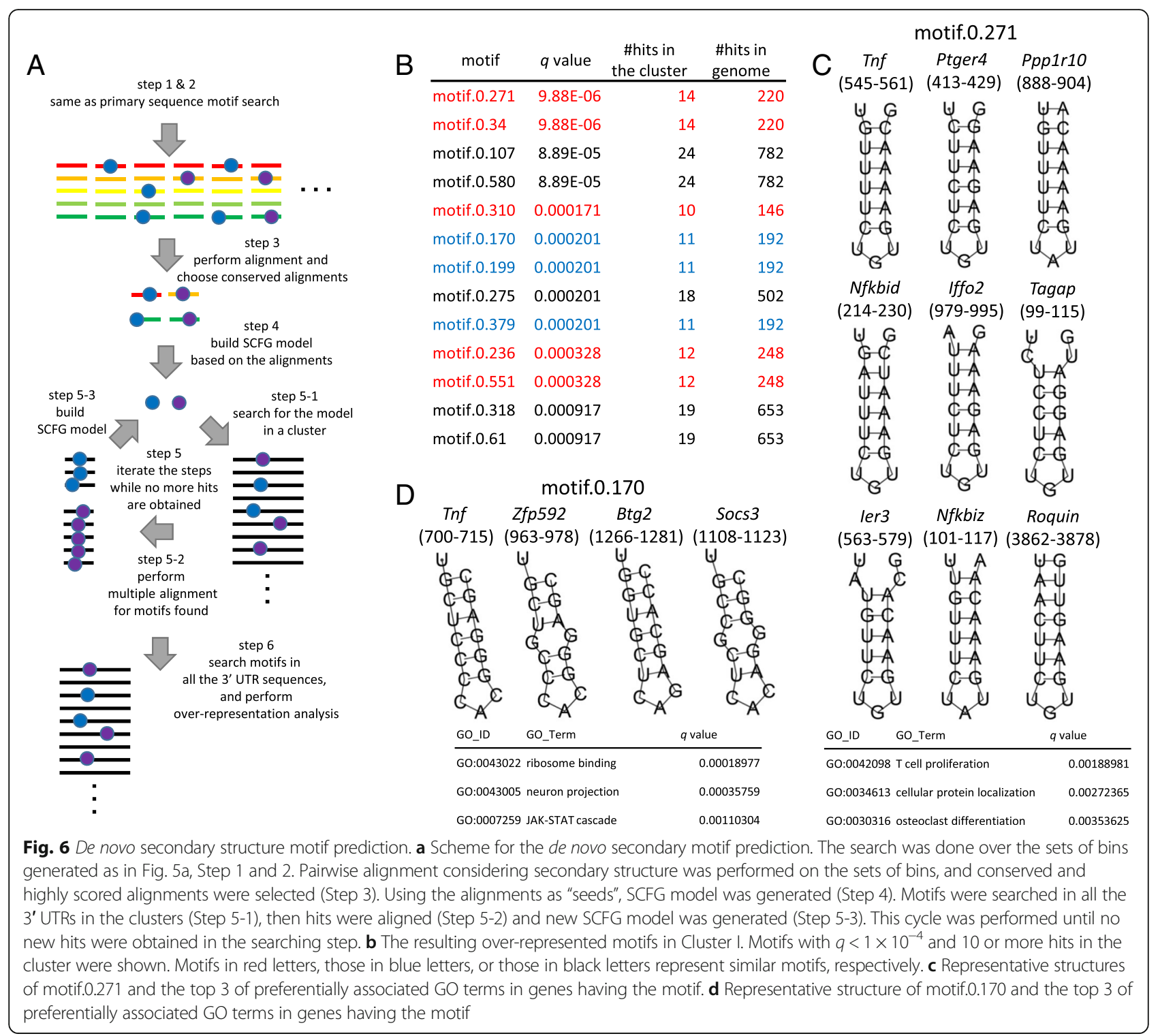

between these studies, the reported range of half-life is consistently from half an hour to 8-30 h, with median values of around 1.5 to several hours. While many of such studies focused to estimate degradation rates of genes in a single condition, we aimed to identify the change of degradation rates as a time course after stimulating cells. Although we lacked replicates in our data due to resource limitation, we attempted to circumvent the limitation of quantitative accuracy of the estimated degradation rates by a qualitative approach based on clustering. Namely, we first classified the degradation time courses and then calculated degradation rates for the resulting clusters of time courses. Despite the limitation, the approach led to results consistent with those previous studies. Our result showed that range of halflife is from around a quarter of hour to $24 \mathrm{~h}$ with median of $3.8 \mathrm{~h}$, indicating reasonable estimation of the RNA degradation kinetics with our procedure.

It has been suggested that degradation of mRNA of genes depends on their cellular functions [36]. Our result confirmed that mRNAs of house-keeping genes are degraded slowly, while those of genes involved in transcriptional regulation and immune signaling, and those of genes encoding cytokines are degraded quickly. Moreover, since our data contains dynamics of gene expression after LPS stimulation, we could reveal associations between expression patterns and degradation kinetics. For example, genes with immediate changes (both induction and repression) in their expression level after LPS stimulation are degraded quickly. In one of the preceding studies, similar to ours, Rabani et al. [5] measured changes of the half-lives of RNAs after LPS 
stimulation at genome-wide level. Although they only reported gene expression and degradation kinetics of relatively early time points (up to $3 \mathrm{~h}$ after stimulation), they also concluded that immediately and transiently induced genes have high degradation rates. Hao and Baltimore reported consistent results on a smaller set of selected genes [35]. Together, we have shown that degradation kinetics of genes correlate with their physiological function and pattern of gene expression level changes.

Some of aforementioned studies utilized ActD chase experiments for measuring degradation as we did in the current study, while others utilized chemical labeling of RNAs with nucleotide analogs [45]. Tani et al. [8] showed that ActD slowed down the degradation of a large fraction of non-coding RNAs. Indeed, we found many genes, including non-coding RNAs, were classified as "stable". Furthermore, in our procedure, the degradation rates of many genes could not be calculated. Together, it is possible that the distribution of degradation rates in cells not perturbed with ActD is different from the current result. Nevertheless, we observed a general consistency with former results on a genome-wide level as discussed above.

Immune cells induce or repress many genes after stimulation. RNA degradation is suggested to be important for controlling this drastic change in expression [39]. Several RNA cis element and RNA-binding proteins have been found to be involved in the process, but our general understanding remains poor. One reason is that the identification of regulatory RNA motifs is very challenging. The prediction of RNA motifs is therefore of great interest. Typically, motif prediction starts from a set of sequences of which all or a large fraction are expected to share a common motif. Most RNA motif prediction approaches are designed for cases where such prior information is available. Therefore, identification of such RNA motifs has required particular RNA-binding protein specific information obtained by using RIP-seq data [37, 38, 41, 43]. Instead, in the current study we first performed pairwise alignment considering both sequence and structural similarity. We built SCFG models based on the alignments and iteratively refined the models within a cluster of interest. This procedure can successfully find motifs even if they are not abundant in the cluster, and thus it is advantageous for the current case in which genes in a cluster share degradation kinetics patterns but do not necessarily share a common motif. By using this procedure, we started from a classification of genes based on degradation patterns and found known functional RNA motifs, and also predicted new candidate motifs, without prior information of binding proteins, although further experimental verification is needed to ensure the function of the motifs.

The successfully identified model of Roquin-and Regnase-1-binding motifs, which was build based on a cluster of interest, also identified the stem loop motif in genes in other clusters, such as Nfkbiz and Roquin, suggesting that the model generated by the procedure has generality for finding targets at a genome-wide level. However, the same model failed to identify some of known target genes of the RNA-binding proteins, such as $I l 6$ and Zc3h12a/Regnase-1 [34, 37, 38]. Since the current procedure builds models by an iterative search-alignment-build cycle in a cluster, it is presumable that the motif model is biased by sequences in the cluster. Alternatively, this finding might suggest the existence of some variety in the motifs recognized by Roquin and Regnase- 1 .

\section{Conclusion}

The current study presents a comprehensive map of patterns of RNA degradation kinetics and indicates that the map facilitated de novo RNA motif prediction. We found strong association between degradation patterns and expression dynamics. Based on the degradation patterns we could find, not only known functional sequence and secondary structure motifs, but also unknown motifs in 3' UTR RNA sequences. The result implied existence of post-transcriptional controls different from that by known pairs of RNA-binding protein and RNA motif. The analysis scheme presented in this study is applicable to other types of genome-wide degradation data and will contribute to elucidating the biology of gene expression control, especially at the post-transcriptional level.

\section{Additional files}

Additional file 1: Tag counts and quantile-normalized RPKM values. (XLSX 14403 kb)

Additional file 2: Supplementary Methods. (PDF 514 kb)

Additional file 3: Pattern recognition of degradation kinetics. Time course data in each of 16 clusters with the mean of the core data of the cluster (black dashed line) and fitting curve (red line) were plotted and shown. The clusters are in the descending order of degradation rates calculated from the fitting, from top, left to right, to bottom. (PDF 443 kb)

Additional file 4: Ranks of degradation speed and clusters. (XLSX 924 kb)

Additional file 5: Scatter plot of alignment scores vs alignment lengths obtained from Cluster I. Scores and lengths of conserved alignments from Cluster I were plotted and shown. (PDF $238 \mathrm{~kb}$ )

Additional file 6: Multiple alignment of sequences found with each motif. Sequences found by searching with the motifs were aligned by Infernal and shown in Stockholm format. Refseq ID of the origin of the sequence, positions of the 3' UTR in the mRNAs of the Refseq ID, and positions of the sequence found by motif searching was shown in the left column. Common secondary structure (SS_cons) and consensus sequence (RF) are also shown. (PDF $214 \mathrm{~kb}$ )

\section{Acknowledgements}

The authors thank A. Yoshimura, E. Kurumatani, Y. Kimura, K. Imamura, K. Abe and T. Horiuchi for technical assistance; M. Ogawa for secretarial assistance; Laboratory of System Immunology in IFReC for computational resource. This work was supported by the Japan Society for the Promotion of Science through Funding Program for World-Leading Innovative R\&D on Science and Technology (FIRST Program) to Y. K., Y. S. and S. A., and by a grant from the Cell Science Research Foundation to Y. K. 


\section{Declarations}

This article has been published as part of BMC Genomics Volume 17 Supplement 13, 2016: 15th International Conference On Bioinformatics (INCOB 2016). The full contents of the supplement are available online at https:// bmcgenet.biomedcentral.com/articles/supplements/volume-17-supplement-13.

\section{Funding}

Publication charges for this article have been funded by a grant from the Cell Science Research Foundation to Y. K.

\section{Availability of data and materials}

The tag sequences in FASTQ format generated by RNA sequencing were deposited at DDBJ (accession number DRA004766)

\section{Authors' contributions}

YK, AV and ST conceived the research. YK performed experiments and computational analysis. AV and ST helped with computational analysis. YS performed RNA-seq experiments. SA helped with experiments. YK, AV and ST wrote the paper. All the authors checked and approved the manuscript.

\section{Competing interests}

The authors declare that they have no competing interests.

\section{Consent for publication}

Not applicable.

\section{Ethics approval and consent to participate}

Not applicable.

\section{Author details}

'Quantitative Immunology Research Unit, WPI Immunology Frontier Research Center, Osaka University, 3-1 Yamada-oka, Suita, Osaka 565-0871, Japan. ${ }^{2}$ Immuno-Genomics Research Unit, WPI Immunology Frontier Research Center, Osaka University, 3-1 Yamada-oka, Suita, Osaka 565-0871, Japan. ${ }^{3}$ Laboratory of Host Defense, WPI Immunology Frontier Research Center, Osaka University, 3-1 Yamada-oka, Suita, Osaka 565-0871, Japan. ${ }^{4}$ Department of Computational Biology and Medical Sciences, Graduate School of Frontier Sciences, The University of Tokyo, Kashiwa 277-8561, Japan.

\section{Published: 22 December 2016}

\section{References}

1. Heng TS, Painter MW, Immunological Genome Project C. The immunological genome project: networks of gene expression in immune cells. Nat Immunol. 2008;9(10):1091-4.

2. Consortium EP. An integrated encyclopedia of DNA elements in the human genome. Nature. 2012:489(7414):57-74.

3. Dolken L, Ruzsics Z, Radle B, Friedel CC, Zimmer R, Mages J, Hoffmann R, Dickinson $P$, Forster $T$, Ghazal $P$, et al. High-resolution gene expression profiling for simultaneous kinetic parameter analysis of RNA synthesis and decay. RNA. 2008;14(9):1959-72.

4. Rabani M, Levin JZ, Fan L, Adiconis X, Raychowdhury R, Garber M, Gnirke A, Nusbaum C, Hacohen N, Friedman N, et al. Metabolic labeling of RNA uncovers principles of RNA production and degradation dynamics in mammalian cells. Nat Biotechnol. 2011;29(5):436-42.

5. Rabani M, Raychowdhury R, Jovanovic M, Rooney M, Stumpo DJ, Pauli A, Hacohen N, Schier AF, Blackshear PJ, Friedman N, et al. High-resolution sequencing and modeling identifies distinct dynamic RNA regulatory strategies. Cell. 2014;159(7):1698-710.

6. Schwanhausser B, Busse D, Li N, Dittmar G, Schuchhardt J, Wolf J, Chen W, Selbach M. Global quantification of mammalian gene expression control. Nature. 2011;473(7347):337-42.

7. Sharova LV, Sharov AA, Nedorezov T, Piao Y, Shaik N, Ko MS. Database for mRNA half-life of 19977 genes obtained by DNA microarray analysis of pluripotent and differentiating mouse embryonic stem cells. DNA Res. 2009;16(1):45-58.

8. Tani H, Mizutani R, Salam KA, Tano K, ljiri K, Wakamatsu A, Isogai T, Suzuki Y, Akimitsu N. Genome-wide determination of RNA stability reveals hundreds of short-lived noncoding transcripts in mammals. Genome Res. 2012;22(5):947-56.
9. Liu Y, Beyer A, Aebersold R. On the dependency of cellular protein levels on mRNA abundance. Cell. 2016;165(3):535-50.

10. Anderson P. Post-transcriptional regulons coordinate the initiation and resolution of inflammation. Nat Rev Immunol. 2010;10(1):24-35.

11. Kafasla P, Skliris A, Kontoyiannis DL. Post-transcriptional coordination of immunological responses by RNA-binding proteins. Nat Immunol. 2014;15(6):492-502.

12. Yiakouvaki A, Dimitriou M, Karakasiliotis I, Eftychi C, Theocharis S, Kontoyiannis DL. Myeloid cell expression of the RNA-binding protein HuR protects mice from pathologic inflammation and colorectal carcinogenesis. J Clin Invest. 2012;122(1):48-61.

13. Lu JY, Sadri N, Schneider RJ. Endotoxic shock in AUF1 knockout mice mediated by failure to degrade proinflammatory cytokine mRNAs. Genes Dev. 2006;20(22):3174-84.

14. Taylor GA, Carballo E, Lee DM, Lai WS, Thompson MJ, Patel DD, Schenkman DI, Gilkeson GS, Broxmeyer HE, Haynes BF, et al. A pathogenetic role for TNF alpha in the syndrome of cachexia, arthritis, and autoimmunity resulting from tristetraprolin (TTP) deficiency. Immunity. 1996:4(5):445-54.

15. Masuda K, Ripley B, Nishimura R, Mino T, Takeuchi O, Shioi G, Kiyonari H, Kishimoto T. Arid5a controls IL-6 mRNA stability, which contributes to elevation of IL-6 level in vivo. Proc Natl Acad Sci U S A. 2013;110(23):9409-14.

16. Pratama A, Ramiscal RR, Silva DG, Das SK, Athanasopoulos V, Fitch J, Botelho NK, Chang PP, Hu X, Hogan JJ, et al. Roquin-2 shares functions with its paralog Roquin-1 in the repression of mRNAs controlling $T$ follicular helper cells and systemic inflammation. Immunity. 2013;38(4):669-80.

17. Matsushita K, Takeuchi O, Standley DM, Kumagai Y, Kawagoe T, Miyake T, Satoh T, Kato H, Tsujimura T, Nakamura H, et al. Zc3h12a is an RNase essential for controlling immune responses by regulating mRNA decay. Nature. 2009:458(7242):1185-90.

18. Konig J, Zarnack K, Luscombe NM, Ule J. Protein-RNA interactions: new genomic technologies and perspectives. Nat Rev Genet. 2011;13(2):77-83.

19. Zambelli F, Pesole G, Pavesi G. Motif discovery and transcription factor binding sites before and after the next-generation sequencing era. Brief Bioinform. 2013;14(2):225-37.

20. Yamashita $R$, Sathira NP, Kanai A, Tanimoto K, Arauchi T, Tanaka Y, Hashimoto S, Sugano S, Nakai K, Suzuki Y. Genome-wide characterization of transcriptional start sites in humans by integrative transcriptome analysis. Genome Res. 2011;21(5):775-89.

21. Langmead B, Salzberg SL. Fast gapped-read alignment with Bowtie 2. Nat Methods. 2012;9(4):357-9.

22. Kim D, Pertea G, Trapnell C, Pimentel H, Kelley R, Salzberg SL. TopHat2: accurate alignment of transcriptomes in the presence of insertions, deletions and gene fusions. Genome Biol. 2013;14(4):R36.

23. Rodriguez A, Laio A. Clustering by fast search and find of density peaks. Science. 2014;344(6191):1492-6.

24. Sugiura N. Further analysts of the data by akaike's information criterion and the finite corrections: Further analysts of the data by akaike's. Commun Stat Theory Methods. 1978;7(1):13-26.

25. Pavesi G, Mauri G, Pesole G. An algorithm for finding signals of unknown length in DNA sequences. Bioinformatics. 2001:17 Suppl 1:S207-14.

26. Nishida K, Frith MC, Nakai K. Pseudocounts for transcription factor binding sites. Nucleic Acids Res. 2009:37(3):939-44.

27. Grant CE, Bailey TL, Noble WS. FIMO: scanning for occurrences of a given motif. Bioinformatics. 2011;27(7):1017-8

28. Sundfeld D, Havgaard JH, de Melo AC, Gorodkin J. Foldalign 2.5: multithreaded implementation for pairwise structural RNA alignment. Bioinformatics. 2016;32(8):1238-40.

29. Durbin R, Eddy SR, Krogh A, Mitchison G. Biological sequence analysis: probabilistic models of proteins and nucleic acids. Cambridge: Cambridge University Press; 1998.

30. Nawrocki EP, Eddy SR. Infernal 1.1: 100-fold faster RNA homology searches. Bioinformatics. 2013;29(22):2933-5.

31. Gruber AR, Lorenz R, Bernhart SH, Neubock R, Hofacker IL. The Vienna RNA websuite. Nucleic Acids Res. 2008;36(Web Server issue):W70-4.

32. Brooks SA, Connolly JE, Rigby WF. The role of mRNA turnover in the regulation of tristetraprolin expression: evidence for an extracellular signalregulated kinase-specific, AU-rich element-dependent, autoregulatory pathway. J Immunol. 2004;172(12):7263-71.

33. Frevel MA, Bakheet $T$, Silva AM, Hissong JG, Khabar KS, Williams BR. p38 Mitogen-activated protein kinase-dependent and -independent signaling of 
mRNA stability of AU-rich element-containing transcripts. Mol Cell Biol. 2003;23(2):425-36.

34. Iwasaki H, Takeuchi O, Teraguchi S, Matsushita K, Uehata T, Kuniyoshi K, Satoh T, Saitoh T, Matsushita M, Standley DM, et al. The IkappaB kinase complex regulates the stability of cytokine-encoding mRNA induced by TLR-IL-1R by controlling degradation of regnase-1. Nat Immunol. 2011;12(12):1167-75.

35. Hao $S$, Baltimore $D$. The stability of mRNA influences the temporal order of the induction of genes encoding inflammatory molecules. Nat Immunol. 2009;10(3):281-8.

36. Yang E, van Nimwegen E, Zavolan M, Rajewsky N, Schroeder M, Magnasco M, Darnell Jr JE. Decay rates of human mRNAs: correlation with functional characteristics and sequence attributes. Genome Res. 2003;13(8):1863-72.

37. Leppek K, Schott J, Reitter S, Poetz F, Hammond MC, Stoecklin G. Roquin promotes constitutive mRNA decay via a conserved class of stem-loop recognition motifs. Cell. 2013;153(4):869-81.

38. Mino T, Murakawa Y, Fukao A, Vandenbon A, Wessels HH, Ori D, Uehata T, Tartey S, Akira S, Suzuki Y, et al. Regnase-1 and Roquin regulate a common element in inflammatory mRNAs by spatiotemporally distinct mechanisms. Cell. 2015;161(5):1058-73.

39. Ivanov $\mathrm{P}$, Anderson P. Post-transcriptional regulatory networks in immunity. Immunol Rev. 2013;253(1):253-72.

40. Beisang D, Bohjanen PR. Perspectives on the ARE as it turns 25 years old. Wiley Interdiscip Rev RNA. 2012;3(5):719-31.

41. Sedlyarov V, Fallmann J, Ebner F, Huemer J, Sneezum L, Ivin M, Kreiner K, Tanzer A, Vogl C, Hofacker I, et al. Tristetraprolin binding site atlas in the macrophage transcriptome reveals a switch for inflammation resolution. Mol Syst Biol. 2016;12(5):868

42. Masuda K, Ripley B, Nyati KK, Dubey PK, Zaman MM, Hanieh H, Higa M, Yamashita K, Standley DM, Mashima T, et al. Arid5a regulates naive CD4+ T cell fate through selective stabilization of Stat3 mRNA.J Jxp Med. 2016;213(4):605-19.

43. Mazan-Mamczarz K, Kuwano Y, Zhan M, White EJ, Martindale JL, Lal A Gorospe M. Identification of a signature motif in target mRNAs of RNAbinding protein AUF1. Nucleic Acids Res. 2009;37(1):204-14.

44. Gorodkin J, Stricklin SL, Stormo GD. Discovering common stem-loop motifs in unaligned RNA sequences. Nucleic Acids Res. 2001:29(10):2135-44.

45. Tani H, Akimitsu N. Genome-wide technology for determining RNA stability in mammalian cells: historical perspective and recent advantages based on modified nucleotide labeling. RNA Biol. 2012;9(10):1233-8.

46. Moré JJ. The Levenberg-Marquardt algorithm: implementation and theory. In: Numerical analysis. Berlin: Springer; 1978. 105-16.

\section{Submit your next manuscript to BioMed Central and we will help you at every step:}

- We accept pre-submission inquiries

- Our selector tool helps you to find the most relevant journal

- We provide round the clock customer support

- Convenient online submission

- Thorough peer review

- Inclusion in PubMed and all major indexing services

- Maximum visibility for your research

Submit your manuscript at www.biomedcentral.com/submit

C) Biomed Central 